\section{SDI an imperfect shield says congressional agency}

Washington

THE Reagan administration's plans for a ballistic missile defence (BMD) have once again come under attack from the congressional Office of Technology Assessment (OTA), which released a critical report last week.

The report says that relatively simple countermeasures by the Soviet Union could foil a large-scale ballistic missile defence system, and that neither the Pentagon nor its contractors have "adequately addressed" the prospect that such a system would have to operate in the face of the "mutual occupation of space by weapons of comparable capability".

The complexity of the Strategic Defense Initiative (SDI) is its major weakness, the OTA report claims, and the system might never be shown to function properly because of the inability to test it adequately. In some of its strongest wording, the report claims that there would be "a significant probability . . . that the first (and presumably only) time the BMD system were used in a real war it would suffer a catastrophic failure".

A summary of the current report was leaked to the press in April (see Nature 332, 767; 1988), but its full findings have only now been made public after two years of study and more than eight months of haggling with the Pentagon over whether parts of it could be declassified.

Because of continued differences between OTA and the Pentagon, the three final chapters - which deal explicitly with potential Soviet countermeasures - were omitted in the unclassified version, much to OTA's displeasure. Of the negotiations with the Pentagon, the report says, "OTA found the wheels of bureaucracy to run very slowly - when they turned at all".

The report's several findings include the following:

- Given optimistic estimates, such as "extraordinarily fast rates of research, development, and production", a limited BMD system might be technically deployable by the year 2000 .

- The decision to deploy a limited BMD system which could destroy "anywhere from a few up to a modest fraction" of attacking Soviet missiles, would face the threat of quick obsolescence by Soviet counter-measures and would (at least to some degree) commit the United States to a system whose ultimate feasibility remains uncertain.

- The question of how well the system's software might work in a real battle situation would "always be irresolvable" which would clearly affect the confidence that the United States military could reasonably place in the system's effectiveness when under fire.

The Pentagon, in a three-page response to the report, criticized OTA's findings as "unduly pessimistic". In particular, the Pentagon cited the report's conclusions about the potential for software problems as reflecting "opinion rather than real analysis".

A key premise of OTA's argument is that large-scale software systems, such as the long-distance telephone system, become dependable only after extensive use and modification. In contrast, such extensive testing would not be possible for a system which could only be used only in a nuclear war.

Despite the Pentagon's criticism, the OTA report is widely seen as one of the most thorough assessments of SDI so far made. Although most of its findings are

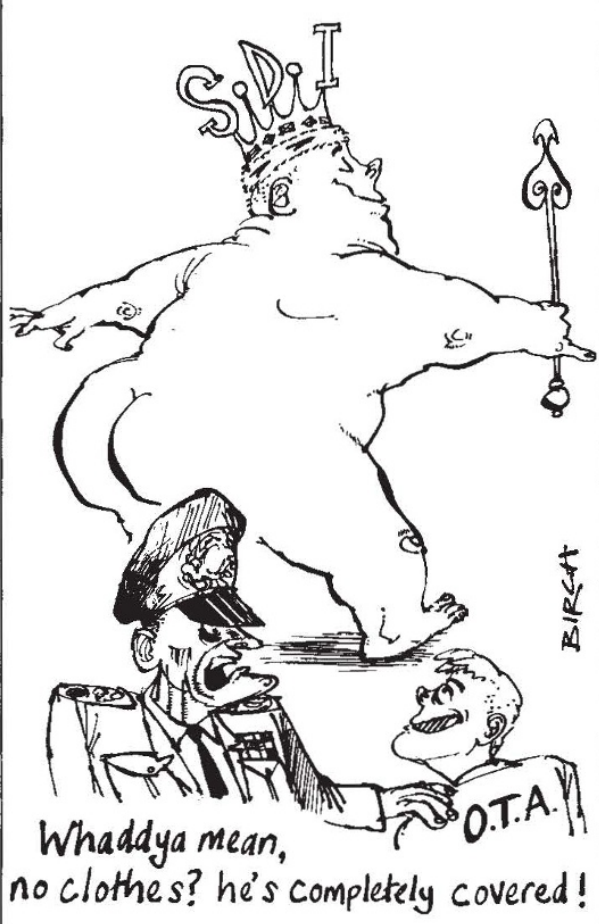

not new, the report has been praised for the diversity of its advisory panel, which included representatives from defence contractors, academic institutions and government, and has been lent further credence by the extensive access that the authors had to classified material during the preparation of the report.

Stephen Meyer, professor at the Massachusetts Institute of Technology and a member of the report's advisory panel says, "this study should play a particularly important role as a primer on the issue for the next Congress and Administration".

Seth Shulman
Engineering lab next on UK privatization agenda

\section{London}

THE British government's recently declared policy of shifting the financial burden of 'near market' research away from the public purse has been dramatically demonstrated with the announcement last week that the National Engineering Laboratory in East Kilbride, Scotland, is up for sale. The laboratory is one of three research establishments run by the Department of Trade and Industry (DTI). Bids for the laboratory, whose value and potential profitability the government has apparently not yet assessed, have been invited, with a deadline of 22 July. The suddenness of the announcement, by Trade Secretary Lord Young, and its implications for future employment prospects, provoked angry criticisms from representatives of the laboratory's workforce of 620 .

The move has also sparked a political row, with allegations of impropriety in the way the sale is being handled. Earlier this year, a small private research firm, British Hydromechanics Research Association, was given permission by the DTI for a limited study of the laboratory's investment potential. The civil service union representing the laboratory's workforce and the local member of parliament want to know why such permission was granted in advance of the announcement of the sale.

The 40-year-old laboratory is involved in a wide range of advanced engineering research, including bioreactors, offshore engineering and wind energy. Of its annual budget of $£ 21$ million, $£ 16$ million comes direct from the government.

The DTI says that the laboratory is a prime candidate for privatization given that around three-quarters of its research is of direct relevance to industry.

The other three DTI research establishments are the Laboratory of the Government Chemist, which provides consultancy advice and studies based on chemistry, with special expertise in analytical chemistry, mainly for the public sector, and monitors the DTI's biotechnology activities; the Warren Spring Laboratory, which undertakes applied research and provides research services for industry, government and local authorities on a wide variety of process industry and environmental problems, including air and oil pollution, measurement and abatement; and the National Physical Laboratory, which provides measurement standards and calibration services.

The government wants these laboratories to remain in the public sector, but to limit the amount of work they do for private industry. Simon Hadlington 